\title{
iBeacon-based Indoor Positioning Systems for Airports
}

\author{
Prachi Singh \\ Student of Bharati Vidyapeeth's \\ College of Engineering \\ (GGSIPU) \\ New Delhi - 110063
}

\author{
Manav Jain \\ Student of Bharati Vidyapeeth's \\ College of Engineering \\ (GGSIPU) \\ New Delhi - 110063
}

\author{
Lakshya Rawal \\ Student of Bharati Vidyapeeth's \\ College of Engineering \\ (GGSIPU) \\ New Delhi - 110063
}

\author{
Preeti Nagrath \\ Assistant Professor \\ Bharati Vidyapeeth's College of Engineering (GGSIPU) \\ New Delhi - 110063
}

\begin{abstract}
To help travelers find their gate or luggage file is a pressing issue for airports. This article introduces an iBeacon based indoor airport positioning system. It first analyzes the advantages of iBeacon over traditional indoor positioning technologies; and then designs the airport indoor positioning system based on the three-tier Internet of Things architecture to provide courier service to customers. Finally, the shortest distance algorithm, Floyd, is used to recommend passengers the next boarding or baggage handling process. Experience has shown that indoor airspace positioning for airports can be achieved through the system.
\end{abstract}

\section{Keywords}

Indoor Positioning System, Floyd, iBeacons, Internet of Things , Low Power Bluetooth

\section{INTRODUCTION}

Although there are floorboards and signs on the floors of most airports, many travelers are still worried that they spend a lot of time finding the boarding or baggage claim area. In recent years, with the development of computer technology and short-range wireless communication technology, the concept of intelligent travel [1] has come into being. Smart Travel refers to the use of state-of-the-art technology-Internet of Things [2], to enable interaction between travelers, airport staff, airline companies and flight areas, and ultimately to achieve computerization.

We can offer indoor positioning for flights through the advanced concept and smart travel technology, improving their flight experience. At present, the technology of indoor positioning is becoming more and more perfect. It is often used in large and medium sized malls and famous. At present, indoor positioning technology [3] is increasingly perfect.

Museums, but rarely used in airports [4-5]. Indoor positioning is via $\mathrm{Wi}-\mathrm{Fi}$, Bluetooth or Radio Frequency Identification technology [6]. Wireless LAN can achieve the goal of positioning, monitoring and tracking the target in a wide range. The self-localization of network nodes is the basis and prerequisite for most applications.

Bluetooth technology locates an object by measuring signal strength. He has some merit. The biggest one is the low volume of the device, which facilitates integration into PDA, $\mathrm{PC}$ and mobile phone. So, its popularization is easier. But he has some disadvantages. First, Bluetooth devices and equipment are expensive. Second, the Bluetooth system in the complex space environment is unstable and prone to being disturbed by a noisy signal. Radio Frequency Identification (RFID) technology uses radio frequency to achieve the goal of detection and positioning through bidirectional contactless data communication.

On the one hand, it has far-reaching benefits of transmission, low cost, and the acquisition of location information in milliseconds. On the other hand, it has the disadvantages of short effect distance and lack of communication ability. In addition, it is difficult to be integrated into other systems. International Journal of Smart Home Vol. 9, No. 7 (2015) 162 Copyright (C) 2015 SERSC. In this paper, iBeacon- based indoor positioning systems for hospital are introduced. It has merits of both the Internet of Things and the mobile Internet and achieves the goal of positioning within hospitals [7]. It has the advantages of low energy consumption, fast response and accurate positioning, making it a great convenience for patients.

\section{BEACON TECHNOLOGY}

iBeacon is the Apple technology that is based on the microlocalization and interaction of a mobile device in the physical world. QR code technology or alternatively NFC technology can be seen to use this technology for its development. Bluetooth Low Energy standard is used by iBeacon which is comprised in the 4th version of Bluetooth.

Sometimes the terms Bluetooth Smart, Bluetooth LE, BLE and simply BLE are used. This is a technology developed by Nokia (originally called Wibree technology, BLE was standardized in 2010) and unlike previous versions of Bluetooth, much lower consumption is typical of BLE. Similarly, the way the device announces its existence to other devices is the opposite of what it is in the original Bluetooth Classic. With BLE, a device can transmit a display packet without being paged from the master device (the central device). With this communication model, it is possible to build BLE transmitters or iBeacons energy-efficient to Apple.

Specific information within a defined radius and at regular intervals is sent by the small Bluetooth Device known as the iBeacon. Once a smartphone enters the area near any iBeacon the iBeacon detects the device and then the sharing of appropriate information can take place. Power consumed by the iBeacon is low since it is a BLE device hence it can work with its set of batteries for 2 years. Of course, battery life depends on transmitter output (TX power) and ad interval settings. 
iBeacon technology is taken over by dealers. A visitor with a BLE-enabled smartphone can be informed about special offers, discounts, information, etc. depending on its position or the proximity of a beacon. He finds similar things in museums and exhibition halls.

Beacons work as a radio transmitter that has a range of 10 to 30 meters indoor.. The advantages of an iBeacon are: They are economical (from $\square 200$ to $\square 2000$ can be installed with minimal effort, determine an exact position up to one meter and are supported by many operating systems and devices. The new BLE (Bluetooth Low Energy) standard is also very energy efficient. Tags can be used for client-based or server-based applications. With the beacons it is possible to recognize the current ground.

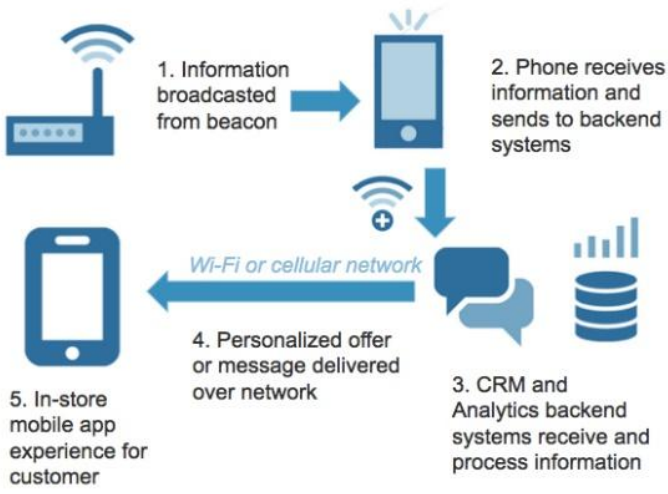

Fig 1: If necessary, the images can be extended both columns

It is not feasible to integrate multimedia content, so tags only send their identifiers (which in the case of the iBeacon protocol are divided into three values: UUID, Major, Minor) and energy information signal.

\section{REVIEW}

\subsection{Usability and Reachability}

How does an iBeacon work? The principles are quite straight forward. Underneath the silicon case is a small ARM computer which is combined with the BLE smart connectivity module which is connected to battery. Nordic Semiconductor's small board runs the Estimate firmware, the low-level software installed on the tags. So, they can figure out a way to respond correctly. While the CPU's processing power and the amount of memory available to it is limited, it is quite sufficient to handle tasks such as processing of sensor data and tag identification encryption for enhanced security

measures.

Whereas when it comes to the finer side of beacon there is a short wire which arrives from CPU: the antenna. It diffuses electromagnetic waves which are in a range of certain length and frequency i.e. $2.4 \mathrm{GHz}$ radio waves. When you open a beacon, it is notable that this antenna has nothing in common with that an antenna of an old television or radio. And the reason is not because it is much smaller but, it's crooked and looks like a bent rod. There is a specific purpose for this. The electromagnetic field generated by a straight wire is in the shape of a donut - the waves do not propagate with the same force in all directions, leaving empty areas behind. The best solution is to change the shape of the antenna. The desired result is to get a good spherical field. However, this is not feasible considering real conditions, so there was a lot of scientific data to determine the good shape of this thread.
There are many books on the working of antenna, and we also experiment to estimate beacons with an antenna that provides the strongest and most reliable field.

Tags use BLE technology for smart communication. This is the latest version of energy efficiency optimized Bluetooth standard, which allows you to transfer only minor packets of data. The extreme load of a Bluetooth 4.2 package is 257 byte which is not enough to integrate multimedia content, which is why the tags send only their IDs, which in the iBeacon protocol are split into three categories namely UUID, Major, Minor and information about the signal strength, which is important for a close smartphone to calculate the proximity. The range and stability are two factors of beacon which are dependent upon: the frequency and the diffusion power. The signal does not transmit continuously - it flashes instead. The adequate interval that it takes the time between each flashing. The more frequently it flashes, the more reliable the signal detection. In fact, mobile phones are not looking for continuous beacons.

They also do this at a certain frequency, and to make it even more complex, this frequency may depend on the state of the phone (locked or unlocked). The type of operating system and device also plays a role. When the phone is active, scans are very often performed. When it's locked in your pocket for a few minutes, it begins to save its battery by limiting the number of Bluetooth analytics.

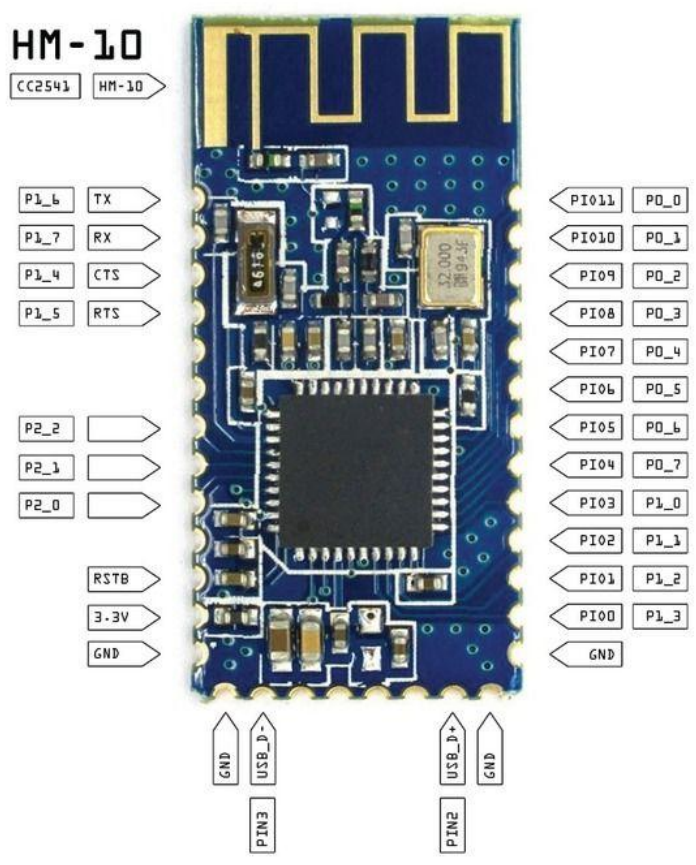

Fig 2: HM-10 module

HM-10 module is a BLE 4.0 based module. This has a motherboard which means that we can program it with rx, and that we also can use FTDI chip to send AT commands via serial connection.

\subsection{Shortest Distance Measurement}

To calculate the shortest distance between two beacons dynamically, instead of fixating latitude and longitude. We used data of signals and their proportionality to the distance between them.

This can be seen when we plot a graph between signal power and beacon on a graph to predict values based on 
one another. The power is measured in $\mathrm{dBm}$ which is explained in detail in a later section.
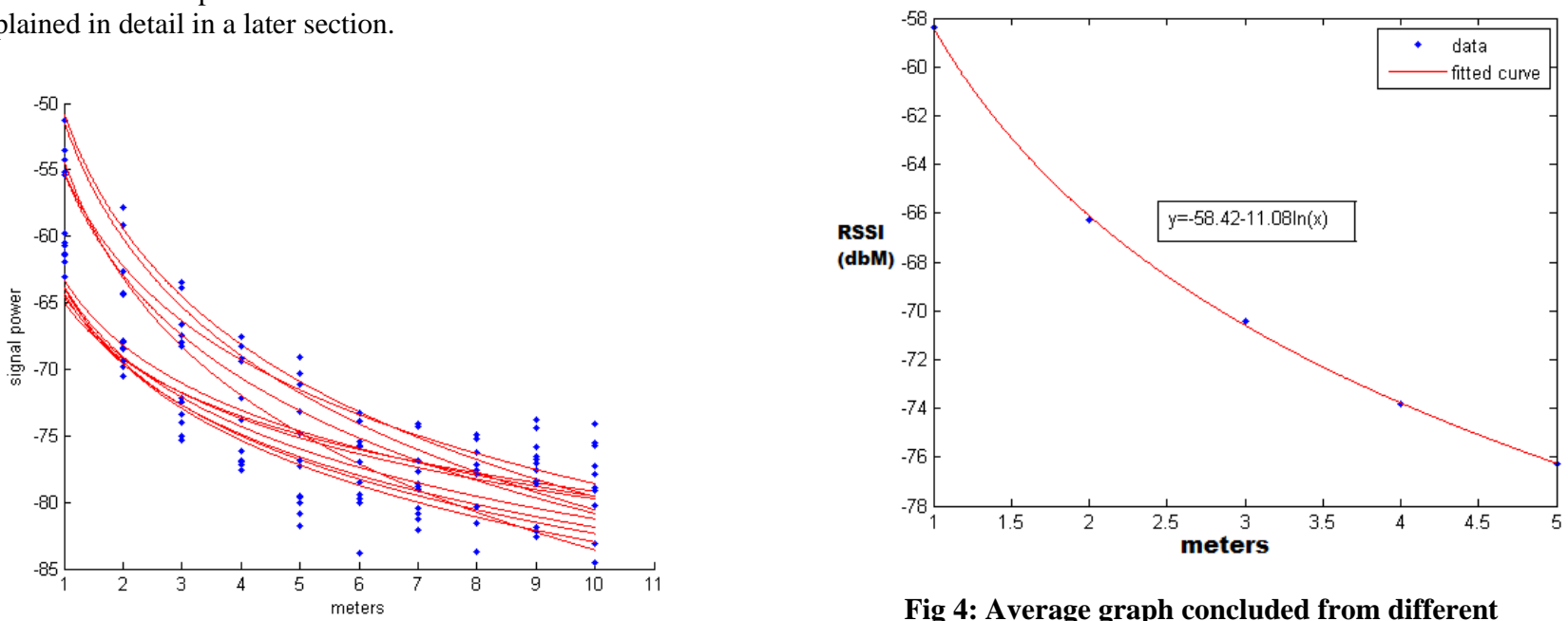

Fig 3: Graph plots for different devices

Fig 4: Average graph concluded from different observations

Table 1. Observation Data

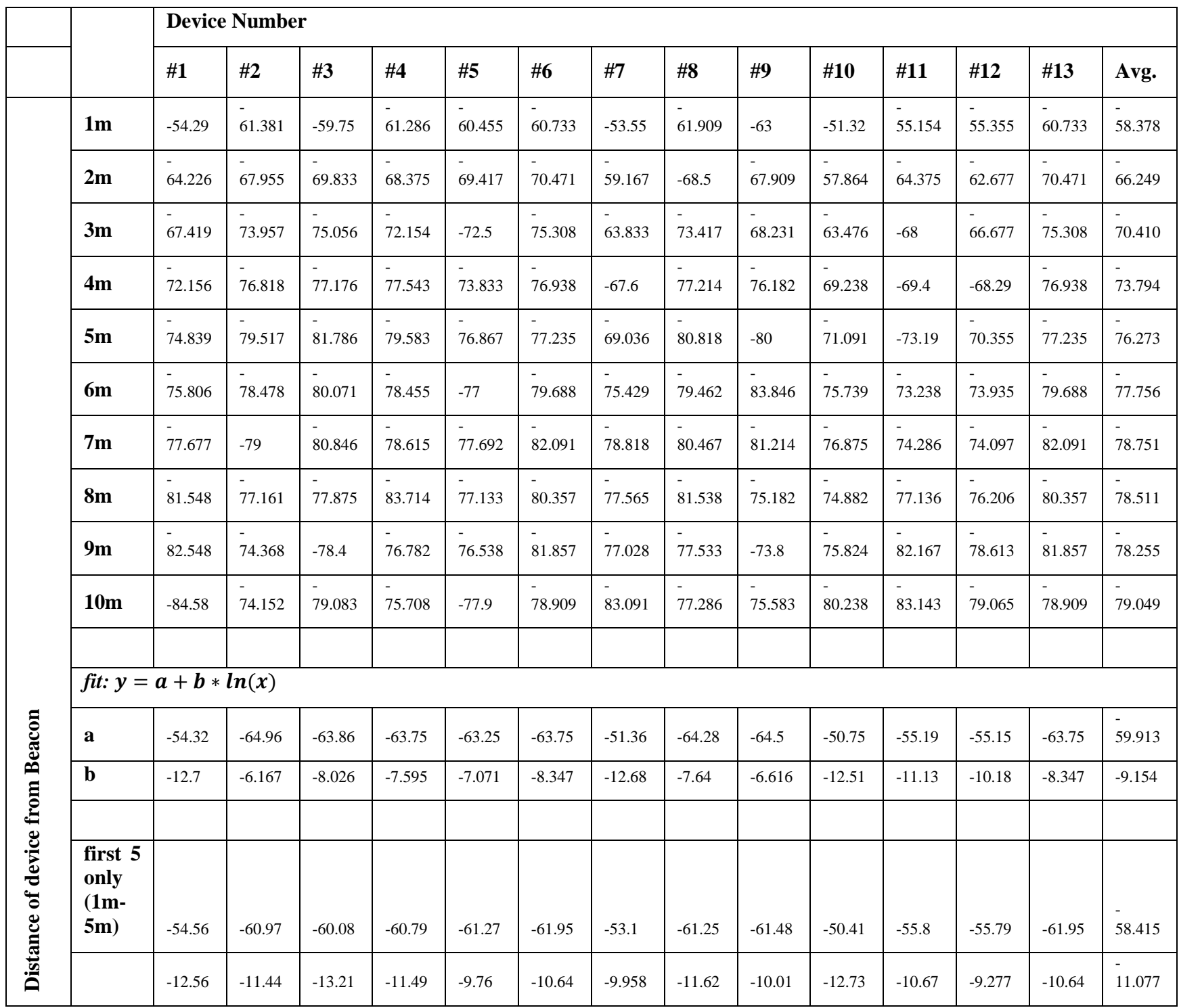




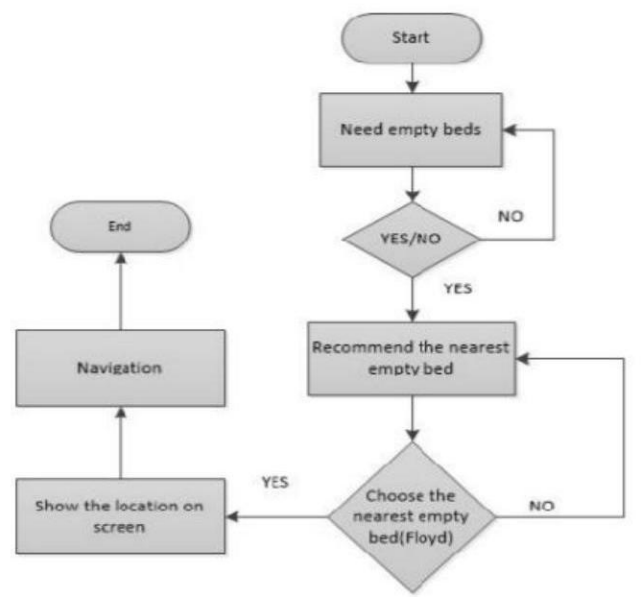

Fig 5: Flowchart for algorithm

\subsubsection{Shortest Path Algorithm}

According to our paper the position of each beacon is relatively stationary. So, we create a backend API that will contain the distance of each adjacent beacon from the current beacon and then we just must find the closest beacon by using Floyd Warshall shortest path algorithm The Floyd algorithm uses the idea of dynamic programming. Shortest path between $x$ and $y$ (our two vertices) is found using this algorithm. That is, all the nodes are used as intermediate points to find the minimum value.

The improved algorithm is as follows:

Step 1. Layout of iBeacons extracted from the API will be taken as an undirected graph. Starting from beacon that is close to our traveler, if it is adjacent to another one, the weight between them is $\mathrm{d}$, and if not, the weight is taken as infinite.

Step 2. For each pair of iBeacons $\mathrm{x}$ to $\mathrm{y}$, whether there is an iBeacon $\mathrm{k}$ that makes the distance from $\mathrm{x}$ to $\mathrm{k}$ to $\mathrm{y}$ shorter than the route known is figured out.

$d_{i j}^{(k)}=\left\{\begin{array}{c}w_{i j}, \quad k=0 \\ \min \left(d_{i j}^{k-1}, d_{i k}^{k-1}+d_{k j}^{k-1}\right), \quad k \geq 1\end{array}\right.$

If there is a $\mathrm{k}$ like that, update when flyers open function interface of navigation, the client will get the current position of the user from server according to ID sent by the iBeacon. After information about the user's position is received, it will be displayed on plane map. By doing this we will help the travelers navigate through the whole airport by following the optimal path provided by our application by using this algorithm.

\subsection{2 dBm (Unit of performance)}

$\mathrm{dBm}$ is a convenient way of measuring performance. $\mathrm{dBm}$ is corresponds to yen time $\log$ of active power ratio to 1 milliwatt.

\section{The exact formula is}

$P(\mathrm{dBm})=10 \times \log 10(P(W) / 1 \mathrm{~mW})$

Where $P(\mathrm{dBm})=$ power expressed in $\mathrm{dBm}$
$P(W)=$ the absolute power measured in watts

$m W=$ milliwatts

$\log 10=$ access base 10

According to this formula, the power in $\mathrm{dBm}$ of 1 watt is 30 $\mathrm{dBm}$. Increment of $3 \mathrm{dBm}$ is about twice the effective power of a signal. The sign of the $\mathrm{dBm}$ also holds significance for example the value $-65 \mathrm{dBm}$ is less strong than the value $40 \mathrm{dBm}$.

It is something like when to owe Rupees 65 to someone than Rupees 40. The signal strengths for mobile networks are always negative $\mathrm{dBm}$ values because the transmitted network is not strong enough to deliver positive $\mathrm{dBm}$ values. The values you specify are like a 5-bar network in GSM, UMTS, or LTE, so you should have no problems due to network strength.

\section{SCOPE}

Beacons are rarely used but does have a very useful implementation in indoor navigation. Our project can be extended for various indoor establishments malls, hospitals, airports, and big complexes like exhibitions and museums. Our review paper expands on the possibility of dynamically locating beacons which makes it more flexible in order to adapt to the various scenarios mentioned above.

\section{CONCLUSION}

Beacons are a very simple piece of technology that can be used by building simple applications and frameworks to widespread there use into mainstream consumer base. The smartphone spectra in current demographic shows a predictable trend in signal distribution which can be mapped and used by developers.

\section{REFERENCES}

[1] B. Michelberger, "iCare: Intelligent Medical Information Logistics", Proceedings of International Conference on Information Integration and Web-based Applications \& Services. ACM, (2013).

[2] H. Kopetz, "Internet of things", Real-time systems, Springer US, (2011), pp. 307-323.

[3] R. Mautz, "Overview of current indoor positioning systems", Geodezija ir kartografija, vol. 35, no. 1, (2009), pp. 18-22.

[4] M. B. Kjæ rgaard, "Indoor positioning using GPS revisited", Pervasive Computing, Springer Berlin Heidelberg, (2010), pp. 38-56

[5] R. Tesoriero, "Using active and passive RFID technology to support indoor location-aware systems", Consumer Electronics, IEEE Transactions on vol. 54, no. 2, (2008), pp. 578-583

[6] R. Mautz, "Overview of current indoor positioning systems", Geodezija ir kartografija, vol. 35, no.1, (2009), pp. 18-22.

[7] Ghose, A. Goldfarb and S. P. Han, "How is the mobile Internet different? Search costs and local activities", Information Systems Research, vol. 24, no .3, (2012), pp. 613-631. 